NIOSH

FACT SHEET

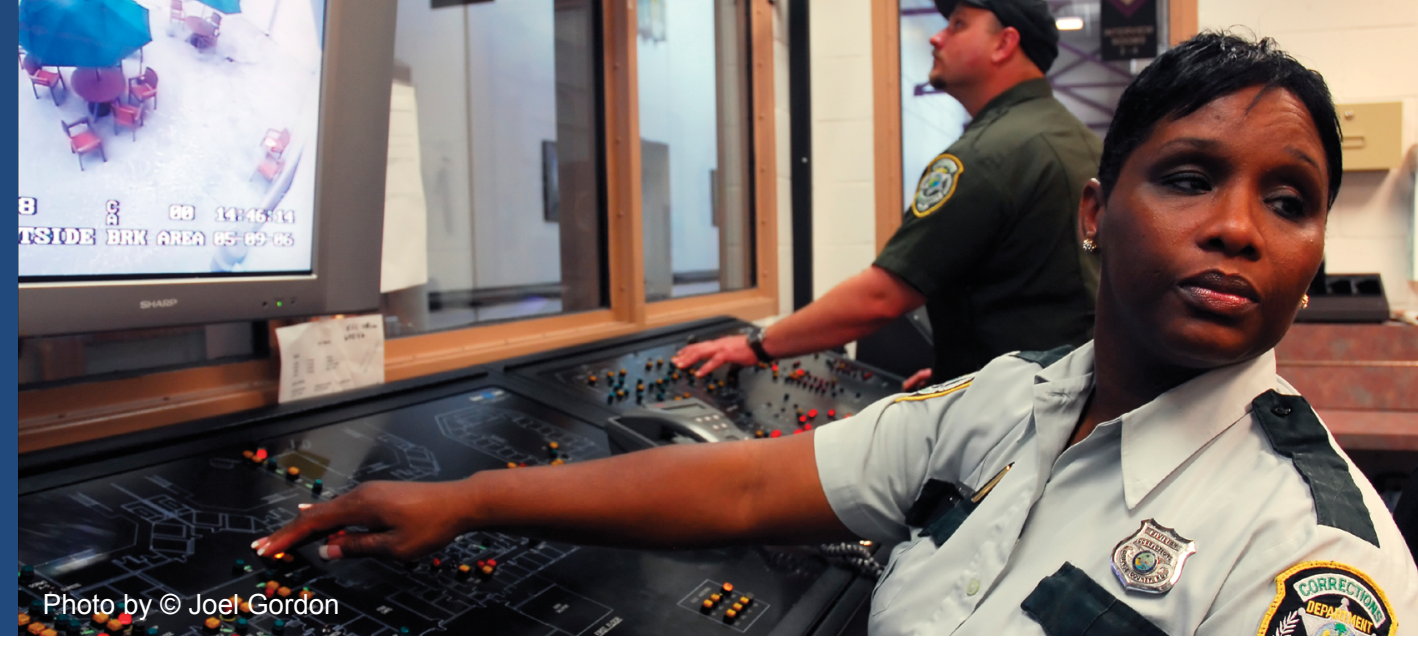

\title{
Managers: Protect Correctional Staff from MRSA
}

\section{Overview}

This fact sheet provides information for managers in correctional settings (including wardens, administrators, risk managers, and supervisors) on how to prevent the spread of MRSA (methicillinresistant Staphylococcus aureus).

\section{Why is it Important to Prevent MRSA?}

MRSA is a potentially dangerous type of staph bacteria. MRSA infections are resistant to treatment by certain antibiotics. Although the infection may start as a mild skin lesion, it can become serious, even fatal.

MRSA outbreaks have occurred at several correctional facilities. Two factors contributing to this are the higher prevalence of MRSA among inmates compared to the general public and crowded living conditions.

\section{Preventing MRSA Saves Money!}

Taking steps to prevent infections among inmates and staff should save money compared to treating them. Miami-Dade County Corrections implemented an infection control plan in 2007, reducing occupational infections and saving over $\$ 93,000$ in workers' compensation costs from 2007 through 2010.*

\footnotetext{
*Miami-Dade Corrections and Rehabilitation Department Infectious Disease Group. Miami-Dade Corrections and Rehabilitation 2010 Annual Progress Report of the Infectious Disease Group.
}

\section{Background on MRSA}

MRSA usually spreads by direct contact with infected skin. It can also spread by touching materials or surfaces that had contact with the infection, including the drainage (pus).

MRSA lesions are often mistaken for spider bites, pimples, or other minor skin problems. They may start as a bump that is red, swollen, painful, warm to the touch, full of pus, or accompanied by a fever.

A healthcare provider should promptly examine skin sores with any of these features among staff or inmates, so they can be diagnosed and treated. This will help keep the infection from getting worse or spreading to other people.

\section{Safety Culture}

Show your staff you care about MRSA prevention. Require workers to follow all health and safety procedures, and include this in their job performance evaluations. Train employees at hire as well as periodically. Emphasize methods of prevention in staff meetings, in health and safety bulletins, or through other methods of communication, such as posters, websites, or videos. 


\section{Managers: Protect Correctional Staff from MRSA}

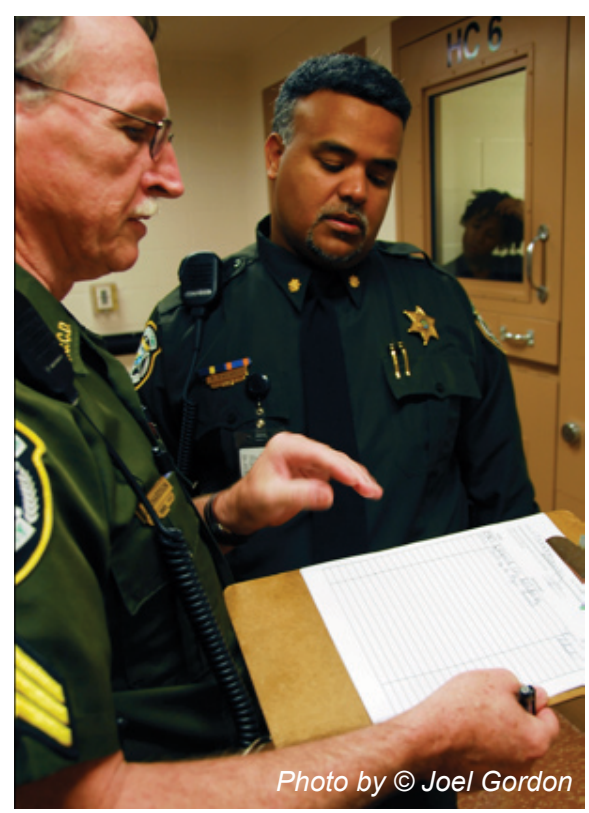

\section{Correctional Standard Precautions}

Educate staff to assume any inmate could be contagious and to take precautions when direct contact with body fluids, non-intact skin, or mucous membranes is anticipated.

\section{Personal Cleanliness}

Handwashing is the most important way to stop the spread of staph/ MRSA. (It also reduces the flu.) Encourage staff and inmates to wash their hands frequently.

Place liquid soap dispensers by all communal sinks used by staff or inmates. It is important that hands are dried with a towel (either paper or individual-use cloth).

When handwashing is not feasible, employees should use alcohol-based hand sanitizer. Keep hand sanitizer in secure locations away from inmates.

Provide wall-mounted or largebottle sanitizer dispensers in secure locations, or suggest that staff carry personal-size bottles of sanitizer with them if they can do so safely.

Inform users that hand sanitizers are not effective when hands are visibly dirty.

Staff should be able to change their clothes if they have significant contamination with body fluids. Allow staff to shower and change as soon as possible after contamination.

Encourage inmates to shower daily and wash their hands frequently. Tell inmates not to share soap or towels with other inmates. Inmates should put on clean clothes often, preferably daily. Inmates should shower after vigorous exercise or heavy sweating.

Instruct inmates not to pop sores themselves.

Tell inmates that tattooing done in prison can spread MRSA.

\section{Facility Cleaning}

Depending on conditions, MRSA can survive on some surfaces for hours, days, or months. That is why cleaning is so important. Keep surfaces that are frequently touched clean. Do not forget about shower handles, faucets, toilets, doorknobs, banisters, exercise equipment, and other surfaces that come into contact with bare skin.

Use cleaners or detergents to remove dust and dirt. Sanitizers reduce but do not eliminate germs on surfaces. EPA-registered disinfectants kill germs. This CDC Web site has more information: http://www.cdc.gov/mrsa/ environment/index.html.

\section{No Sharing of Personal Items}

Educate staff and inmates not to share personal items (clothing, towels, razors, etc.) because this can spread MRSA.

\section{Laundry}

Require staff and inmates to use gloves when handling dirty laundry or when stationed near laundry handling. Handle wet or soiled laundry as little as possible and place it in a leak-proof bag. Consider washing laundry that is soiled with MRSA separately.

Ensure laundry is machinewashed with detergent and machine-dried thoroughly in high heat. Use the warmest water recommended on detergent and clothing labels. Avoid over-filling the washing machine-the items may not get clean, and MRSA may not be removed.

\section{Food Handlers}

Routinely examine inmate food handlers for visible skin infections. Emphasize to all food handlers the importance of immediately reporting all skin infections, no matter how minor.

\section{Screen and Treat Inmates}

Prompt identification of MRSA and appropriate medical treatment are essential elements of control.

To reduce the spread of MRSA, encourage inmates with skin symptoms to seek prompt medical care. Sick visit fees can discourage inmates from seeking prompt medical care. 


\section{Managers: Protect Correctional Staff from MRSA}

Screen inmates for skin infections: (1) on intake, (2) on return from the hospital, and (3) during a MRSA outbreak. For inmates with medical risk factors for MRSA infections (such as diabetes, open wounds, or chronic skin conditions), screen during routine medical evaluations.

Instruct staff to refer inmates with visible or self-reported skin sores to medical services.

Healthcare providers should:

(1) follow appropriate protocols for culturing lesions, (2) review culture results in a timely manner, (3) follow appropriate protocols for prescribing antibiotics, and (4) instruct infected inmates on proper wound care.

Ensure a standard medical screening and treatment protocol is in place and followed. If healthcare is contracted out, be sure the contract spells out how MRSA and suspicious lesions will be evaluated and treated.

\section{Housing Infected Inmates}

Follow Federal Bureau of Prisons or institutional guidelines for housing infected inmates.

\section{Transferring Infected Inmates}

Avoid transferring inmates with skin and soft tissue infections to other facilities until fully evaluated and treated. If transfer must

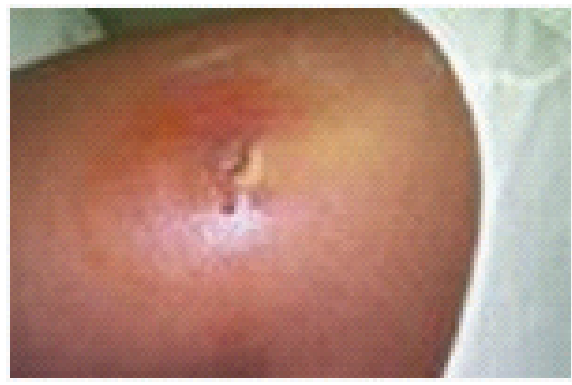
occur, inform partner agencies (while complying with HIPAA*).

*Health Insurance Portability and Accountability Act of 1996

\section{The Most Important Steps to Prevent MRSA}

\section{Safety Culture}

- Demonstrate to your staff that you support MRSA prevention.

\section{Personal Hygiene}

- Encourage frequent hand washing, and provide soap, water, and individual-use towels.

- Provide alcohol-based hand sanitizer for employees' use when hand washing is not feasible.

- Keep hand sanitizer in secure locations away from inmates.

\section{Environmental Sanitation}

- Use cleaners or detergents for routine cleaning of surfaces.

- Use sanitizers as needed to reduce the number of germs on surfaces.

- For surfaces that have drainage from infected skin, clean the surface, and then use an EPA-registered disinfectant to kill the germs.

- Follow directions on the labels, and ensure use of gloves and eye protection as recommended.

\section{Prompt and Appropriate Medical Care}

- Consider waiving inmate sick call fees for skin sores.

- Promptly refer inmates with skin sores to medical services.

- Ensure that facility healthcare providers follow recommended treatment protocols.

- Refer employees with skin symptoms to their personal physician or the department's physician.

\section{Personal Protective Equipment (PPE)}

- Provide and encourage the proper use of PPE, especially gloves.

\section{No Sharing of Personal Items}

- Instruct staff not to share items such as uniforms, clothing, towels, or water bottles.

- Instruct inmates not to share towels, washcloths, clothing, bars of soap, or razors.

\section{Training}

- Frequently educate staff and inmates about staph and MRSA and how to avoid infections. 


\section{Managers: Protect Correctional Staff from MRSA}

Notify escort officers of the inmate's condition, and remind them of infection control precautions. Use disposable restraints when feasible, and decontaminate reusable restraints.

\section{Releasing Infected Inmates}

If inmates are released while infected, provide them with the remainder of their medications whenever possible and instructions to take all the pills. Also provide information on wound care and how to keep the infection from spreading to household contacts.

\section{If a Staff Member is Diagnosed with Staph or MRSA Infection}

Set up a procedure for employees and contractors to notify supervisors if they are diagnosed with staph or MRSA infection.

Since MRSA is known to be transmitted in correctional facilities, in order to halt its spread, investigate whether the infection might have been work-related.

Medical staff should be able to help managers look for links among cases and opportunities for infection prevention.

\section{Work Restrictions for Infected Employees and Inmates}

Unless a healthcare provider says not to, most people with MRSA infections can go to work. It is safe to work with someone who has a MRSA skin infection as long as the wound is kept clean, dry, and covered.

Restrict from work:

- Those with wound drainage that cannot be covered and contained with a clean, dry bandage or who cannot maintain good hygiene practices, until the infection has healed.

- Those with active infections from activities where skin-to-skin contact with the affected skin area is likely to occur, until the infection is healed.

- Food handlers with a lesion containing pus (such as a boil) or infected wound that is open and draining unless it is covered in accordance with the Food Code 2009 (http://www.fda.gov/FoodCode2009).

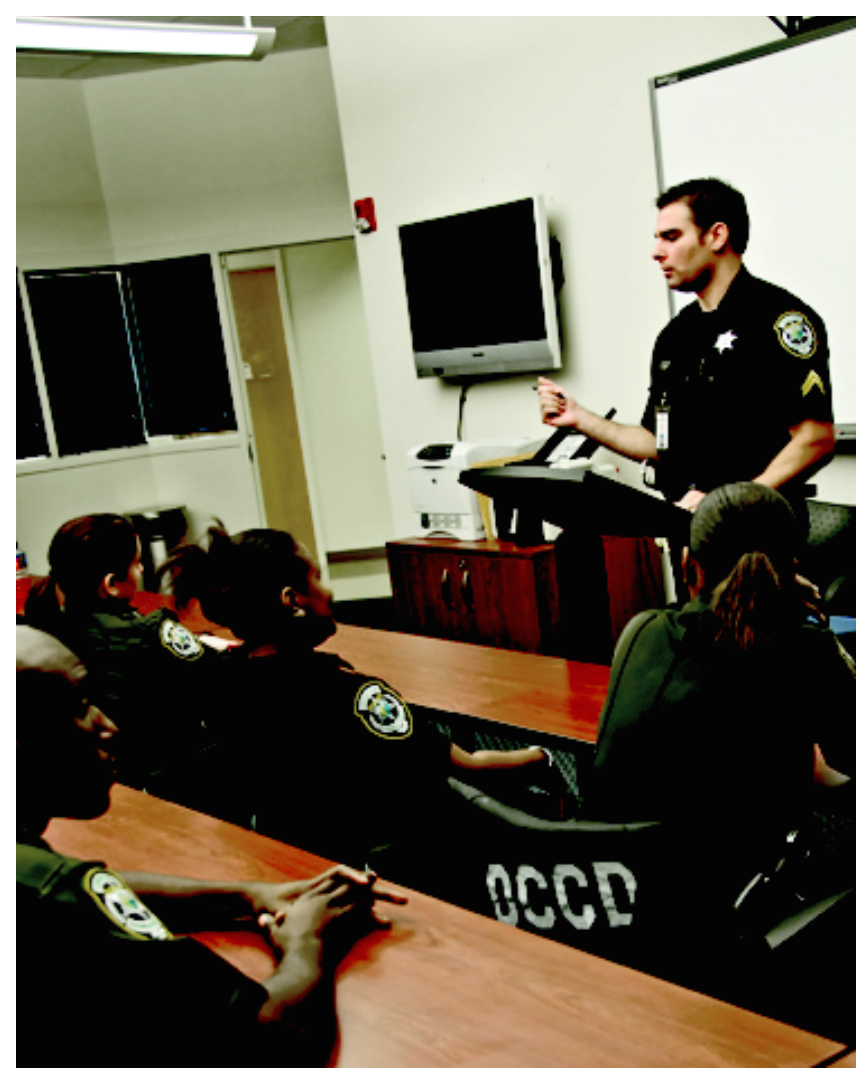

Photo by @ Joel Gordon

\section{Additional Information}

NIOSH: www.cdc.gov/niosh/topics/MRSA CDC: www.cdc.gov/mrsa BOP Guidelines: www.bop.gov/news/PDFs/mrsa.pdf EPA-registered disinfectants:

www.epa.gov/oppad001/list_h_mrsa_vre.pdf

To receive documents or other information about occupational safety and health topics, contact $\mathrm{NIOSH}$ at

Telephone: 1-800-CDC-INFO (1-800-232-4636)

TTY: 1-888-232-6348

E-mail: cdcinfo@cdc.gov

or visit the NIOSH Web site at www.cdc.gov/niosh.

For a monthly update on news at $\mathrm{NIOSH}$, subscribe to $\mathrm{NIOSH}$ eNews by visiting www.cdc.gov/niosh/eNews.

DHHS (NIOSH) Publication No. 2013-120

January 2013

SAFER • HEALTHIER - PEOPLETM 